\title{
FRACTURE MECHANICS OF PLATE DEBONDING
}

\author{
Mithila ACHINTHA Chris BURGOYNE
}

Department of Engineering, University of Cambridge, UK

Keywords: debonding, fracture mechanics, FRP, strain energy

\section{INTRODUCTION}

The enhanced structural performance of reinforced concrete (RC) beams that have been strengthened by externally bonded fibre reinforced polymer (FRP) plates is strongly reliant on the effectiveness of the concrete-FRP interface. Strengthened beams often fail by premature plate debonding [1]; an understanding of the plate debonding mechanism is of the greatest important for the successful application of external FRP plates on RC beams. It is important to know where and when debonding initiates and the influence of parameters such as the plate curtailment location and the plate thickness. Over the last decade much research has focused on the plate debonding failures, but a critical examination shows this problem is far from being solved.

Two modes of plate debonding are experienced in simply-supported externally-strengthened RC beams [1] (Figure 1); one initiates at a crack in the beam mid-span zone and propagates towards the nearest beam end, while the other initiates at a plate end and propagates inwards. The first mode is termed intermediate-crack induced debonding or mid-span debonding and the second mode is plate end debonding. High interfacial stresses are present in the vicinity of an existing crack and at the plate end, which initiate these two debonding modes. The temptation is therefore to compute these interfacial stresses and compare them with interface strength properties to determine the failure loads.

However, the debonding mechanisms of plates glued to concrete structures are proving very difficult to analyse. The interface can be modeled using finite elements but it is contended that this procedure is doomed to failure. A re-entrant corner leads to an infinite stress concentration, so the values returned by a finite element program are governed by the smallness of the elements used, and by unwarranted assumptions about adhesive properties which the analyst is forced to make.

Fracture-mechanics models, such as that due to Hutchinson [2] offer a better alternative. They assume that, since flaws are inevitable in the interface, what matters is whether these flaws can propagate to cause debonding. When an existing flaw extends, the energy needed to form associated new surfaces depends on the interface fracture energy and must be compared with the energy released by the system, which in turn depends on the change of strain energy stored in the system. An essential prerequisite is thus that the energy states of the beam must be known.

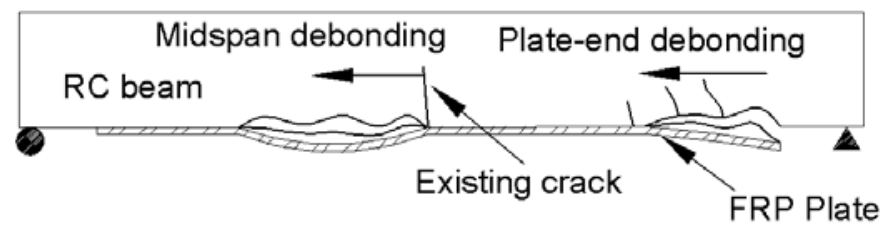

Fig. 1 Two modes of debonding

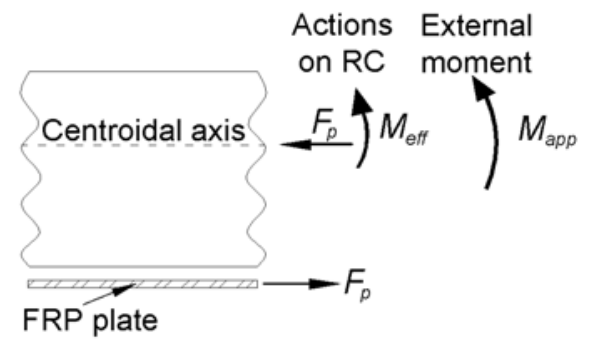

Fig. 2 Actions on longitudinal section

The energy calculations for a concrete beam require knowledge of the moment-curvature relationship. It is well-known that when a RC beam cracks its stiffness does not immediately change to that of a section where the tension concrete can be fully disregarded. Various empirical models, such as Branson's $I_{\text {eff }}$ concept, have been used to model this behaviour [3], primarily with a view to being able to predict the deflections of RC beams to check their compliance with code limits. However, the Branson analysis only covers the case of a cracked-elastic beam in the absence of any axial force.

The main complication inherent to a strengthened beam is that the FRP plate acts as a prestressing element, inducing both force and moment in the RC beam (Figure 2). Most existing models do not cope with this effect, but it is needed for an accurate determination of the strain energy. 
The present work extends the Branson analysis into the inelastic regime, and also takes account of the axial forces.

An overview of the proposed model is presented here, along with examples of how it can be used to determine the strain energy; a detailed derivation of the model together with its validation against test data is published elsewhere [4]. The paper describes how, once the energy state of the beam is known, fracture mechanics concepts can be used to answer the question "Will this existing interface crack extend?" Both modes of debonding are analysed as is the effect of curtailment location on the debonding mode.

\section{FRACTURE-MECHANICS MODEL FOR PLATE DEBONDING}

Since flaws are inevitable in the interface, what matters is whether an existing flaw can propagate. The proposed model first assumes a debonding-crack of known length and orientation. The energy states of the beam are computed, both in this state and when the crack is further extended by a small distance $\delta x$. The energy released from the system due to the crack extension can then be determined from energy conservation concepts; part of this released energy is consumed to create the new fracture surfaces required for crack extension. The extension of the crack will occur only if there is sufficient released energy to create the required new surfaces. Thus, whether an existing debondingcrack will propagate or not can be decided by comparing the possible available energy with the energy that is actually required. The energy needed to form associated new fracture surfaces depends on the interface fracture energy, the determination of which is discussed in section 6 . The present discussion concentrates on the determination of the energy released from the system when an existing debonding crack extends.

\subsection{Energy available for debonding}

The force in the FRP plate $\left(F_{p}\right)$ is chosen in such a way that the relevant compatibility condition between the FRP plate and the RC beam is satisfied [4]. If the FRP plate is perfectly bonded to the beam, then strain compatibility must be satisfied locally between the FRP plate and the strain in the tension fibre of the concrete. If the FRP plate has debonded then the weaker condition has to be satisfied in which the extension of the plate in the debonded region is same as the extension of the tension fibre in the concrete.

Thus, when the debonding-crack extends, $F_{p}$ in the debonded zone may alter to retain compatibility. This change in $F_{P}$ causes alterations in the strain and stress profiles of the relevant RC sections, and hence the energy states alter, releasing some energy. At each boundary where the debonded and the fully-bonded zones are separated, a discontinuity in $F_{p}$ can be expected since the computations are based on two different compatibility conditions. Sharp discontinuities in $F_{p}$ cannot occur so there must be a transition zone where there is some relative slip between the FRP and the beam. The transition zone $F_{p}$ profile depends on the difference between the two $F_{p}$ values in the unbonded and fully-bonded areas. In the fully-bonded region, $F_{p}$ does not change unless the applied load changes, since the more rigorous strain compatibility condition still applies, so it is the change in $F_{p}$ in the unbonded zone that causes variation in the $F_{p}$ profile over the transition zone with a consequent energy release. Expressions to determine the transition zone $F_{P}$ profiles are given in section 5; full details of the derivation are described elsewhere [5].

Sections in both the debonded and the transition zones release energy when the crack extends, which must be summed to obtain the total energy release at the crack tip. For a given section, it is important to know the amount of energy that is available for release.

When a RC beam bends, energy is put into the beam by the loads, some of which is dissipated in the concrete, either in flexural-tension cracking or nonlinear elasticity, and by yielding of the steel reinforcement, whilst the rest is recoverable and stored as strain energy, shown schematically in Figure 3. Thus, for a given section, only the stored strain energy that can be recovered when the beam is completely unloaded is active and will be responsible for the change of the energy state of the section upon debonding. The moment from which the unloading takes place, and the corresponding unloading $M-\kappa$ relations, should be known for the determination of the strain energy.

\section{MOMENT-CURVATURE RELATIONSHIPS}

Any calculation of the stiffness of RC beams at the working load must make some allowance for the tensile stresses in concrete that is partially cracked. A detailed analysis would require knowledge of the exact location of the reinforcement and each of the cracks which is unknowable. For most 
practical purposes it has been sufficient to determine the effective stiffness of the section, or of the beam, using an interpolation formula. That derived by Branson [3] is most commonly used.

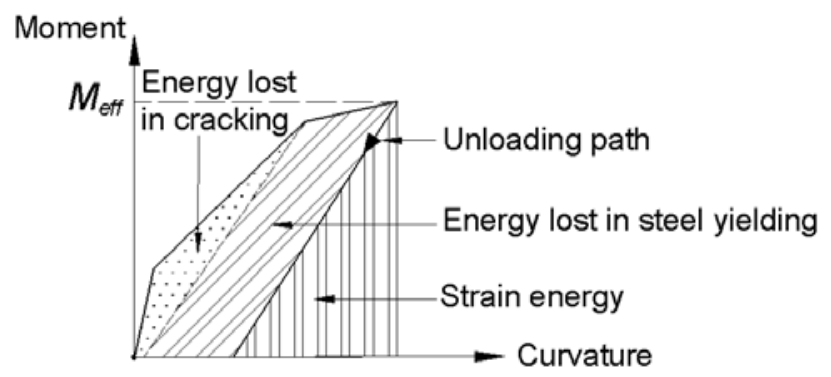

Fig. 3 Energy in flexure

\subsection{Branson's $I_{\text {eff }}$ expression}

Branson derived an expression (Eq. (1)) for the effective stiffness $\left(l_{\text {eff }}\right)$ that indirectly accounts for tension stiffening effects of cracked concrete [3]. The stiffness is interpolated between the uncracked state $\left(I_{u n}\right)$, where the concrete is fully effective in tension, and the fully-cracked state $\left(I_{f c}\right)$ where there is no tension stiffening. The interpolation coefficient ( $K$ in Eqs. (1) and (2)) represents the extent of cracking of the section.

$$
\begin{gathered}
I_{\text {eff }}=K I_{u n}+(1-K) I_{f c} \\
K=\left(M_{c r} / M_{\text {app }}\right)^{4}
\end{gathered}
$$

$M_{c r}$ and $M_{a p p}$ in Eq. (1) are the moments causing first cracking and the externally applied moment respectively. $l_{\text {eff }}$ is taken as $I_{\text {un }}$ when $M_{\text {app }} \leq M_{c r}$. A modified form is used when an average $I_{\text {eff }}$ of the beam is required for deflection predictions. In this case the exponent in Eq. (1) is reduced from 4 to $3 . l_{\text {eff }}$ in Eq. (1) is the effective second moment of area of the equivalent transformed concrete section of modulus $E_{c}$, so curvature of the section $(\kappa)$ can be determined from:

$$
\kappa=M_{\text {app }} /\left(E_{c} I_{\text {eff }}\right)
$$

Equations (1-3) can be used to determine the local curvature of a section and have been widely verified for conventional RC beams; they will now be extended to deal with the more complex problem of beams with external FRP plates.

\subsection{Branson's model for strengthened beams}

In a strengthened section, the FRP plate can be considered as a prestressing element, inducing both force and moment on the original RC section, even when fully bonded. Thus, the RC section is subjected to the action of a compressive force $F_{p}$ (the force in the FRP plate) and an effective moment $\left(M_{\text {eff }}\right.$ ), both acting at the RC section's centroid (Figure 2). Branson's method will need modifying to take account of several factors.

Branson's expression (Eq. (1)) only applies to RC beams subject to pure bending, which can be regarded as a simple couple, so there is no need to define a particular reference axis and no need to worry about the distinction between the centroid and the neutral axis. In the present study the RC beam has to be analysed under a moment and the axial force, so axes need to be defined.

Branson's concepts were primarily concerned with stiffness and are not normally used to determine stresses in the beam, which are assumed to be adequate since separate checks (either permissible stress or section strength) would also be performed. The present method will require the satisfaction of a compatibility condition between the FRP and the concrete. That means that a strong assumption needs to be made about the strains, and hence stresses, in the cross-section, and these will have to be determined from the effective stiffness [4].

Equation (1) was designed for applications where the beam was at the working load, so the stresses everywhere would be relatively low; the materials could be assumed to be linearly elastic. That will not be the case here, since the model is to be applied to sections that are being strengthened to allow them to carry loads that would have caused the original section to fail. Some, at least, of the steel in the section may be yielding and the concrete stresses will be high enough that non-linearity 
should be taken into account. Thus, the objective is to determine the moment-curvature behaviour of a partially-cracked beam when being loaded and unloaded, subject to an applied moment and an axial force, which must satisfy compatibility conditions with the external reinforcement that may be bonded or unbonded, using materials which may be non-linear, all of which must sit within a nonlinear loop in which the force in the FRP plate is unknown.

\subsection{Proposed moment-curvature model for strengthened sections}

As mentioned in section 2.1, the force in the FRP plate $\left(F_{p}\right)$ can only be determined by satisfying the relevant compatibility condition. The equations are set up using an assumed value of $F_{p}$, and a solution of the nonlinear equations is found using a least square method. The section is then reanalysed with the correct value of $F_{p}$ to obtain the actual stress-strain distributions. Full details of the equations and procedure can be found in elsewhere [4].

When the amount of cracking of a RC section increases, tension-stiffening eventually becomes ineffective. In Branson's model (Eq. (1)) the stiffness becomes asymptotic to the fully-cracked state, but never reaches it. That model was intended to represent sections at the working load, and well below yield of the reinforcement. The present model will apply to beams where the loads cause yield of the conventional reinforcement and which rely on the FRP for security; it can be expected that the fully-cracked state will be reached. It will now be assumed that the section is fully-cracked at the moment causing first yielding of the tension steel $\left(M_{y}\right)$ and a slightly modified form of the interpolation factor used in Eq. (1) is proposed:

$$
K=\left(M_{c r} / M_{\text {app }}\right)^{4}\left[1-\left\{\left(M_{\text {app }}-M_{c r}\right) /\left(M_{y}-M_{c r}\right)\right\}^{4}\right]
$$

This expression has the property that $K$ is zero when $M_{\text {app }}=M_{y}$. When the $M_{y}: M_{c r}$ ratio is greater than about 3, which would be the case for most practical RC beams, the difference between the two predictions is negligible and this expression avoids a discontinuity when the section yields [3].

Equation (4) allows the extent of cracking of a partially-cracked section at any given $M_{\text {app }}$ to be represented as a function of $M_{c r}, M_{y}$ and $M_{a p p}$. However, only a part of the corresponding externally applied moment is effective on the RC section, and hence the relevant effective moments should be compared. To avoid unrealistic contributions due to varying eccentricities of the force in the FRP a fixed reference axis will be used for the comparison; the beam centre line is chosen and the corresponding effective moments are denoted as $M_{c r-m i d}, M_{y-\text { mid }}$ and $M_{\text {app-mid }}$ respectively. Thus, the interpolation coefficient used in the present model becomes:

$$
K_{P}=\left(M_{c r-\text { mid }} / M_{\text {app-mid }}\right)^{4}\left[1-\left\{\left(M_{\text {app-mid }}-M_{c r-\text { mid }}\right) /\left(M_{y-\text { mid }}-M_{c r-\text { mid }}\right)\right\}^{4}\right]
$$

At higher strains the concrete is nonlinear and steel could yield, so a cracked-linear elastic analysis is not applicable with strengthened sections. It is possible to define an equivalent elastic stiffness $\left(E l_{e q}\right)$ of a strengthened section for use in place of the elastic stiffness (the product of $E_{c}$ and $\left.l_{\text {eff }}\right)$ used with the Branson's concept:

$$
E l_{\text {eq }}=M_{\text {eff }} / \kappa
$$

where $\kappa$ and $M_{\text {eff }}$ are the curvature and the effective moment on the RC section about its centroid. $\kappa$ of uncracked and fully-cracked sections can be found from the relevant section analysis. $M_{\text {eff }}$ is related to the externally applied moment $\left(M_{\text {app }}\right)$ by Eq. (7).

$$
M_{\text {eff }}=M_{\text {app }}-F_{p} \times\left(h+t_{a}+t_{p} / 2-\alpha\right)
$$

The depth of the RC section, and the thicknesses of the adhesive layer and the FRP plate in Eq. (7) are given by $h, t_{a}$ and $t_{p}$ respectively. The centroidal axis depth is given by $\alpha$, the determination of which can be found in elsewhere [4]. Thus, the respective $E l_{e q}$ of the uncracked and fully-cracked sections can be found. The effective $E l_{e q}$ of a partially-cracked section is then interpolated by:

$$
E l_{\text {eq-eff }}=K_{p} E l_{\text {eq-un }}+\left(1-K_{p}\right) E l_{e q-f c}
$$

The effective equivalent elastic stiffness $\left(E l_{\text {eq-eff }}\right)$ calculated from Eq. (8) is relative to the effective centroid of the section. The moment $\left(M_{\text {eff }}\right)$ about this centroidal axis is then calculated from Eq. (7), and the curvature $(\kappa)$ of the section is finally calculated from: 


$$
\kappa=M_{\text {eff }} / E l_{\text {eq-eff }}
$$

Equation (9) allows the curvature of the section to be determined, from which the strain distribution across the section can be calculated. The deflection profiles of beams can also be found by integration of the curvatures. Comparisons between test data and the present model for some of the test specimens found from the literature show good agreement [4] (see, for example, Figure 4); it is concluded that the proposed $M-\kappa$ model is accurate and reliable and thus can be extended into strain energy computations of strengthened beams.

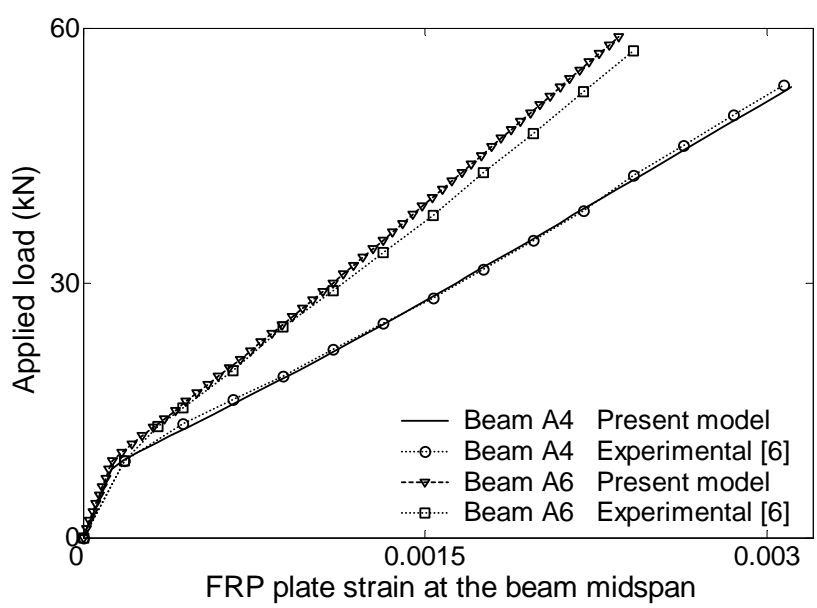

Fig. 4 Strain comparison

\section{STRAIN ENERGY IN A STRENGTHENED SECTION}

As described, the RC beam part of a strengthened section is subjected to an axial force and moment, while the FRP plate is under tension (Figure 2). Therefore, the total strain energy (SE) in a strengthened section consists of three parts; SE in the RC beam due to flexure (flexural SE), SE in the $\mathrm{RC}$ beam due to axial force (axial SE) and SE in the FRP plate (FRP SE).

\subsection{Flexural strain energy}

It is assumed that all the constituents are linear-elastic upon unloading, so the unloading $M-\kappa$ relations are linear irrespective of the moment from which the unloading takes place, although there will usually be some residual curvature if the load is completely removed. Thus, the flexural strain energy available in a beam segment of unit length at a given effective moment ( $\left.M_{\text {eff }}\right)$ is calculated from:

$$
\left.S E_{E}\right|_{\text {flexure }}=\frac{1}{2} M_{\text {eff }} \kappa_{U L}
$$

where $M_{\text {eff }}$ and $\kappa_{U L}$ are the effective moment on the RC section and the corresponding change in curvature upon complete unloading.

\subsection{Axial strain energy}

A strengthened RC beam segment is subjected to a net axial compressive force of magnitude $F_{p}$ at the section's centroid (Figure 2). The axial strain energy in the segment of unit length $\left(\left(S E_{e}\right)_{\text {Axial }}\right)$ can be calculated as:

$$
\left.S E_{E}\right|_{\text {Axial }}=\frac{1}{2} F_{p} \varepsilon_{0}
$$

where $\varepsilon_{0}$ is the change in centroidal strain upon complete unloading, which can be calculated when the strain profile after unloading is known [5].

\subsection{Strain energy in the FRP plate}

The FRP plate is assumed to be linear-elastic so the strain energy in a unit length is: 


$$
\left.S E_{E}\right|_{F R P}=\frac{1}{2} \frac{F_{p}^{2}}{E_{p} A_{p}}
$$

where $E_{p}$ and $A_{p}$ are the elastic modulus and the cross sectional area of the FRP plate respectively.

Now that the energy state of the strengthened beam segment under given external actions can be calculated, energy conservation can be used to determine the energy released from the segment when the existing debonding crack extends [5].

\section{PLATE DEBONDING ANALYSIS}

The proposed model assumes that there must be a debonding crack present of known length at a given location. The energy release rate $\left(G_{R}\right)$ when it extends is calculated and compared with the interface fracture energy $\left(G_{F}\right)$ to decide whether the crack will actually propagate or not. However, the zones of the beam from which energy can be released should first be identified.

\subsection{Energy release zones for plate-end debonding}

When the FRP plate is curtailed at a nonzero moment location, the axial strain difference between the plate end and the concrete immediately adjacent to it (i.e. zero axial strain in the FRP and a nonzero strain in the concrete) causes relative slip between the two adherents in the vicinity of the plate end. Thus, for a section near the plate end, the relevant $F_{p}$ cannot be determined by assuming a unique linear strain distribution across both the beam and the plate, despite the plate still being "bonded" to the beam. This slip and the corresponding shear stresses are assumed to decay in an exponential manner, and are significant only over a transfer zone [5,7]. For a section outside the transfer zone, a linear strain distribution across both the FRP plate and the beam can be assumed because the plate is here fully-bonded.

The externally applied loads on the beam are assumed to remain unchanged during crack extension, so for a fully-bonded section, $F_{p}$ and the strain profiles, and hence the energy state, cannot be altered due to crack extension. Thus, there can be no energy released from the fully-bonded sections. However, the $F_{p}$ profile over the transfer zone is altered by the crack extension and, as described in section 2.1, energy releases are expected.

\subsection{Identification of the energy release zones}

Figure 5 shows a strengthened beam with an assumed plate-end debonding crack $A B$, and the region $\mathrm{BC}$ is the relevant transfer zone. The crack then extends by small distance $\delta x$. Assuming that the transfer zone length is fixed for the given system, the zone DE is the new transfer zone. The plate is unbonded over the zone $A B$ during both stages and hence, neither the strain profiles nor the energy states of the relevant sections are altered by the transformation, and there is no associated energy release. Similarly, there are no energy releases from the sections to the right of $E$ (Figure 5) as those are fully-bonded. The narrow zone BD was initially within the transfer zone, and thus carried some force in the FRP, but after the crack extension the zone has become unbonded. The zone CD is within the transfer zone for both the stages, but the $F_{p}$ profiles have changed. The narrow zone CE has changed from the fully-bonded state to the transfer state. In all three regions the stress distribution has changed so some energy will have been released.

The strain energy calculations of the sections with known $F_{p}$ values are performed as in section 4 , but for the sections with no effective FRP (sections within zone $A B$ before extension and $A D$ afterwards), only the flexural energy must be considered. However, the $F_{p}$ profile within the transfer zone, and the length of the transfer zone, are both required for the $G_{R}$ determination.

A detailed analysis of the transfer zone, taking account of all the material nonlinearities and the tension stiffening effects of cracked concrete is virtually impossible to perform. Linear elastic solutions have been presented, for example that by Täljsten [7], which reveal that the effects of the plate-end slip decay exponentially. It is however contended that within the transfer zone the stress transfer between the RC beam and the FRP plate primarily depends on the stiffness properties of the plate and the interface shear-stress/relative-slip characteristics. Neither the material behaviour nor the presence of flexural cracks in the RC beam have significant effects on the interface stress transfer. Thus, it is believed that the Täljsten analysis [7] adequately represents the stress transfer between the FRP plate and the beam irrespective of the amount of nonlinearity in the concrete. 
The force $\left(F_{p}\right)$ is assumed to vary as:

$$
\left.F_{p-s}\right|_{x=x}=\left.F_{p-b}\right|_{x=x}-\left.F_{p-b}\right|_{x=0} e^{-\lambda x}
$$

where $\left.F_{p-s}\right|_{x=x}$ is the value of $F_{p}$ at a position $x$ from the plate end (but within the transfer zone) while $\left.F_{p-b}\right|_{x=x}$ is the value of $F_{p}$ that would occur at that location if the FRP was fully-bonded.

The associated length scale $\lambda$ is defined as:

$$
\lambda=\sqrt{\frac{G_{a}}{t_{a}} \times \frac{1}{E_{p} t_{p}}}
$$

where $G_{a}$ and $t_{a}$ are the shear modulus and the thickness of the adhesive, and $E_{p}$ and $t_{p}$ are the Young's modulus and the thickness of the FRP plate respectively. This is a slight simplification of the expression determined by Täljsten [7], but comparisons [5] with a large database of beam specimens covering many variations of geometric, loading and material (RC beam, FRP plate and the adhesive) properties, show good correlations with the more accurate Täljsten analysis and also show that a transfer zone length of 30 times the FRP plate thickness is a reasonable approximation; this is used in all subsequent analyses.

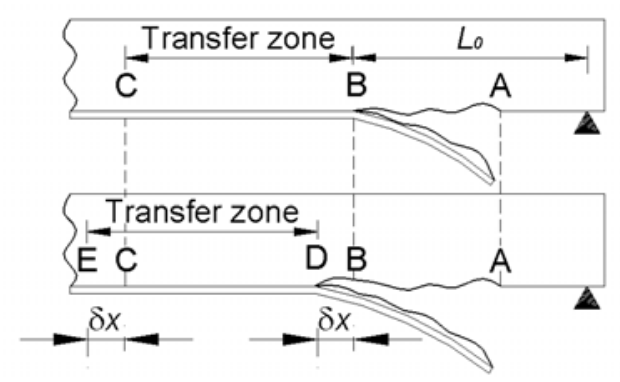

Fig. 5 Energy release zones for plate end debonding (a)

(b)

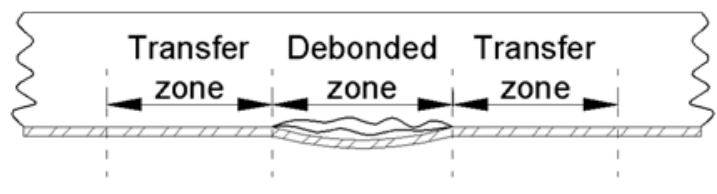

Fig. 6 Energy release zones for mid-span debonding

\subsection{Energy release zones for mid-span debonding}

The mid-span debonding analysis is more complicated than that of the plate end debonding, because there exist two end regions, both fully bonded, separated by a region in which the FRP plate is debonded but still constrained by a compatibility condition (Figure 6).

At each boundary of the debonded zone, the unbonded $F_{p}$ gradually reaches the fully-bonded value. Thus, when the crack extends, the resultant variations in $F_{p}$ over both transition zones and the debonded zone should be considered in the determination of the total energy released. The values of $F_{p}$ in the bonded zone at the boundary and in the unbonded region can be determined in a separate analysis, and the transition between them is assumed to vary exponentially using the same characteristic length $\lambda$ described above. The change in force at the boundary between the two regions can be positive or negative, depending on the circumstances.

\subsection{Energy release rate}

It is now possible to determine the energy release rate when the crack propagates. The zones where energy is being dissipated (the debonded zone and the transition zones) are divided into short segments and the energy released from each segment due to the assumed crack extension is then computed. The total energy release at the crack tip $\left(\Delta E R_{t}\right)$ is obtained by summing the individual energy releases in all segments. Assuming uniform energy release during the crack extension, which is independent of crack length, the energy release per unit area of crack extension $\left(G_{R}\right)$ is:

$$
G_{R}=\Delta E R_{t} /\left(b_{f} \times \delta x\right)
$$

where $b_{f}$ and $\delta x$ are the width of the FRP plate and the horizontal-linear crack extension respectively.

$G_{R}$ can then be compared with the interface fracture energy (i.e. energy required to fracture a unit area of the interface, $G_{F}$ ) to decide whether the flaw will extend or not. If $G_{R} \geq G_{F}$ the flaw will extend causing debonding; if not there is insufficient energy for the flaw to propagate. 


\section{CONCRETE-FRP INTERFACE FRACTURE ENERGY}

It is now necessary to determine $G_{F}$. The propagation of an interface crack takes the path requiring least energy. Experimental evidence confirms that concrete-FRP debonding fractures generally propagate through the concrete just above the interface $[1,8]$. It is however reported that due to poor surface preparation prior to plate bonding or the use of low-strength adhesives, the debonding fractures can propagate along the interface or within the adhesive [8]. With the availability of high-strength adhesives and careful surface preparation techniques, these adhesive and interface failures can be precluded. Thus, the present work concentrates only on the case where the debonding-fractures propagate through the concrete, as this is the actual problem encountered in the industry.

\subsection{Fracture mode of the interface-concrete}

Since the debonding fractures propagate through the concrete, the interface fracture energy $\left(G_{F}\right)$ must be taken as that of the concrete. In two-dimensional space there are three possible failure modes. Mode I, the opening mode, governed by tensile stresses present at the crack tip; Mode II, the sliding mode, governed by shear stresses, or the mixed-mode with both tensile and shear stresses at the crack tip can occur in a body. For a given material, the fracture energies of these different modes will not be the same. In the vicinity of the interface, both normal and shear stresses are inevitable, so it is contended that the debonding fractures in the strengthened beams are governed by mixed-mode effects. However, it is widely accepted that, even under mixed-mode loading conditions, concrete fractures grow locally in a pure Mode I state, and hence the corresponding fracture energy is the pure Mode I fracture energy [9].

\subsection{Fracture energy of concrete}

Even when checking an existing beam, it is very difficult to determine the Mode I fracture energy of the concrete $G_{l c}$, but a reasonable assumed value can be used.

In concrete, the nonlinear fracture process zone (FPZ) ahead of a pre-existing crack tip is relatively large compared to the structural dimensions, and therefore its effects should not be neglected as is done in linear elastic fracture mechanics. Material softening taking place in the FPZ should be taken into account in the estimation of $G_{l c}$. Hillerborg's fictitious crack model [10], which represents $G_{l c}$ as the area under the corresponding softening stress-crack surface separation $(\sigma-w)$ curve, is widely believed to be the best available simple nonlinear concrete fracture model [11]. The exact $\sigma-w$ relations of a given concrete can only be known from direct uniaxial tensile tests. Nevertheless, a few $\sigma-w$ models which describe the softening curve in terms of more readily available parameters of the concrete, based on databases of test results, have been proposed in the literature [12-14]. The corresponding values for $G_{I c}$ for the concrete mix described in section 7 are given in Table 1. Based on these values, $0.15 \mathrm{~N} / \mathrm{mm}$ is taken as the $G_{l c}$ of the concrete used in the debonding analysis presented below.

\section{EXAMPLE}

To illustrate the application of the model, a RC beam, strengthened with a CFRP plate, loaded as shown in Figure 7, has been analysed. The compressive and the tensile strengths of the concrete are assumed to be 35 and $3.7 \mathrm{~N} / \mathrm{mm}^{2}$ and the Young's modulus and the yield stress of steel are assumed as $200 \mathrm{kN} / \mathrm{mm}^{2}$ and $530 \mathrm{~N} / \mathrm{mm}^{2}$ respectively. The shear modules of the adhesive is taken as 4.2 $\mathrm{kN} / \mathrm{mm}^{2}$ and the Young's modulus of the CFRP is $165 \mathrm{kN} / \mathrm{mm}^{2}$. The unstrengthened capacity of the beam is $65 \mathrm{kN}$; after strengthening with CFRP, the failure load should be about $145 \mathrm{kN}$, assuming that debonding does not take place. The failure mode would have changed from an under-reinforced failure before strengthening to an over-reinforced mode afterwards.

\subsection{Plate end debonding}

Figure 8(a) shows the variation in energy release rate $G_{R}$ if debonding occurs at different load levels, for different locations of the plate curtailment position $L_{0}$. If $L_{0}$ is less than about $270 \mathrm{~mm}$, the energy release rate is less than $G_{l c}$ and no plate end debonding would occur before the beam reached its flexural capacity. If $L_{0}$ is greater than $270 \mathrm{~mm}$, it is predicted that premature failure would occur at lower loads. Fig $8(\mathrm{~b})$ shows the failure load against $L_{0}$. A plot such as this can be used to determine how far the plate should extend so that additional external anchoring devices are not needed. 
Table 1 Fracture energy estimations

\begin{tabular}{ccc}
\hline Reference & $\begin{array}{c}\text { Softening } \\
\text { model }\end{array}$ & $\begin{array}{c}G_{F} \\
(\mathrm{~N} / \mathrm{mm})\end{array}$ \\
\hline$[12]$ & bilinear & 0.154 \\
{$[13]$} & bilinear & 0.162 \\
{$[14]$} & polynomial & $0.09-0.16$ \\
\hline
\end{tabular}

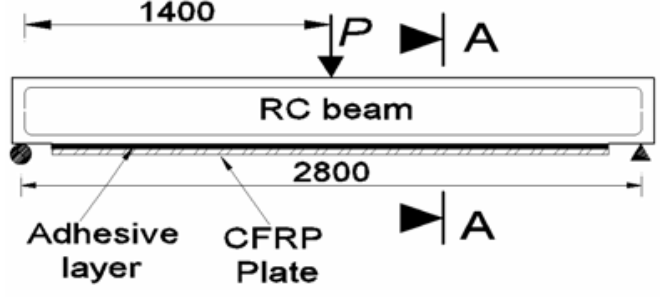

Note: All dimensions in millimetres

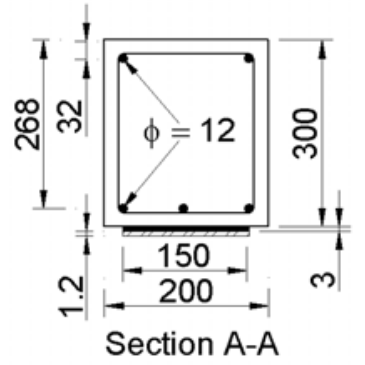

Fig. 7 Specimen dimensions and loading data

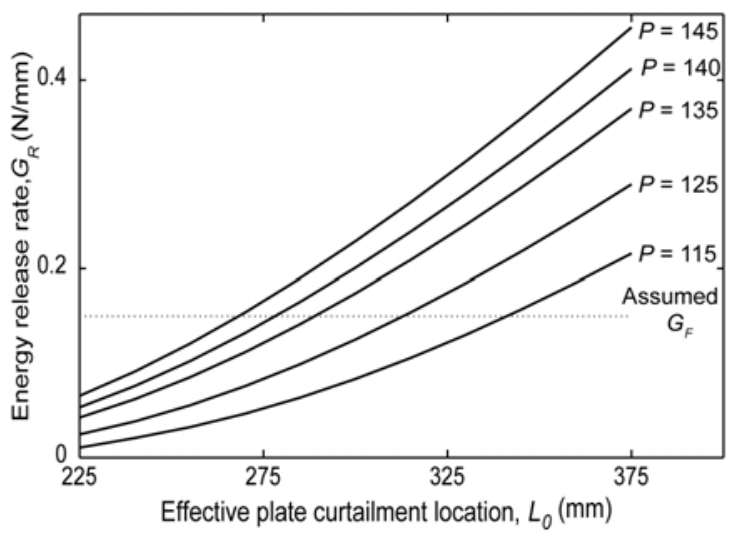

(a) $G_{R}$ for different $L_{o}$ and $P(k N)$

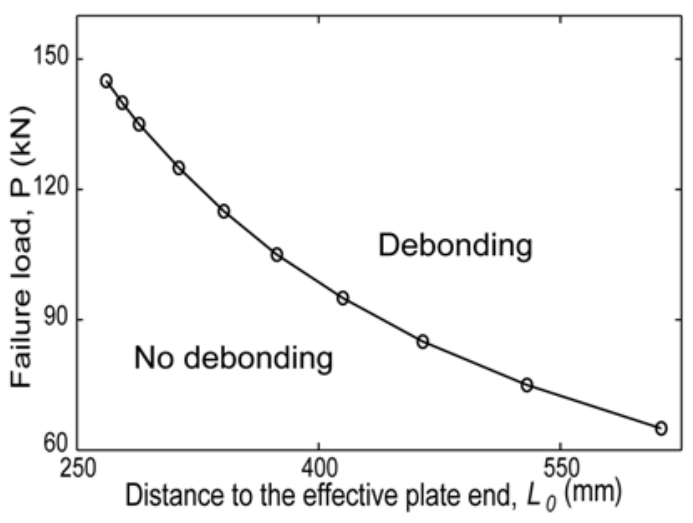

(b) Failure load against $L_{0}$

Fig. 8 Plate end debonding

\subsection{Mid-span debonding}

Figure 9(a) shows the energy release rate for a fracture propagating from an internal crack, one end of which is $100 \mathrm{~mm}$ from the centreline, with a crack length of $l_{d}$. This shows that if the crack length exceeds about $2 \mathrm{~mm}$, debonding would occur towards the nearest end support at a load less than the strengthened capacity of the beam $(145 \mathrm{kN})$. Debonded lengths of this sort of dimension may well be present, and undetectable, in many practical applications. If the initial fracture is longer than this, debonding will take place at lower loads.

The possibility that the fracture might extend in the opposite direction has also been studied (as shown in Fig 9(a) by dashed lines), but these show that more load, or a higher crack length, would be required to propagate in this direction, so it is unlikely to be critical.

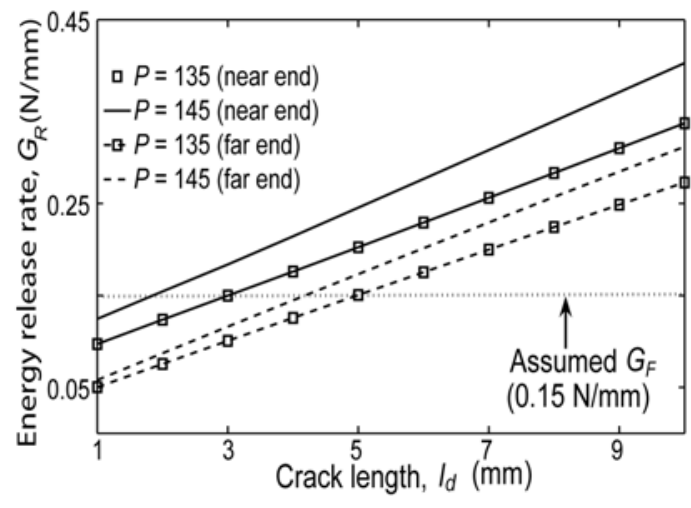

(a) $G_{R}$ for different crack lengths at a fixed crack location

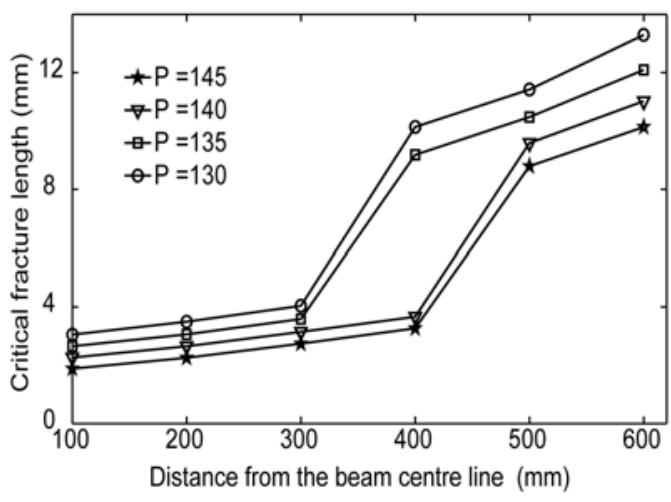

(b) Critical fracture lengths for different crack locations

Fig. 9 Mid-span debonding 
Figure 9(b) shows contour plots of the load at which mid-span debonding will take place against crack length and crack location. Short cracks that start near mid-span (on the left of the diagram), where the sections at the crack, and in the transfer zone, are both fully cracked. If the crack starts further away from the centre-line (to the right of the diagram), the moments reduce and the extent of cracking in the sections is reduced and there is less strain energy that can be released. The effect is that more load, or a longer crack, is needed to cause debonding.

Studies of the detailed results show that, for shorter crack lengths, most of the strain energy release takes place in the transfer zone, because these are relatively long. The results also show that, although the axial strain energy stored in the concrete is quite small (typically about $1 \%$ of the total), the contribution to the release rate can be as high as $20 \%$. These aspects are the objects of further study.

\section{CONCLUSIONS}

The study has shown that the phenomena of plate debonding can be studied by means of a fracture-mechanics approach, which obviates the need for a finite element analysis which would have dubious validity in the presence of infinite stress concentrations.

It has been necessary to produce a modified form of Branson's method to allow the calculation of the beam stiffness when the section is partially cracked and when subjected to an axial load imposed by the FRP plate.

Hutchinson's interface breakdown model has proved to be a very useful tool for the study of the debonding of FRP plates from concrete structures. More work remains to be done to study the importance of the various parameters that influence the result. Comparisons with experimental data in the literature are being undertaken.

\section{REFERENCES}

[1] Leung, C.K.Y., "Delamination Failure in Concrete Beams Retrofitted with a Bonded Plate", Journal of Materials in Civil Engineering, 13, 2, 2001, pp 106-113.

[2] Hutchinson, J.W. and Suo, Z., "Mixed Mode Cracking in Layered Materials", Advances in Applied Mechanics edited by J. W. Hutchinson and T. Y. Wu, 29,1992, pp 63-191.

[3] Branson D.E., "Design Procedures for Computing Deflections", ACl Journal Proceedings, 65, 9, 1968, pp $730-735$.

[4] Achintha, P.M.M. and Burgoyne C.J., "Strain Energy of Beams with Bonded and Unbonded External FRP Reinforcement", 2006, to be submitted to ACI Structural Journal.

[5] Achintha, P.M.M. and Burgoyne C.J., "A Fracture-mechanics model of plate debonding", 2006, to be submitted to Journal of Composites for Construction.

[6] Arduini M., Di Tommaso A. and Nanni A., "Brittle Failure in FRP plate and Sheet Bonded Beams", ACl Structural Journal, 94, 4, 1997, pp 363-370.

[7] Täljsten B., "Strengthening of Beams by Plate Bonding", Journal of Materials in Civil Engineering, 9, 4, 1997, pp 206-212.

[8] Buyukozturk O., Gunes O. and Karaca E., "Progress on Understanding Debonding Problems in Reinforced Concrete and Steel Members Strengthened using FRP Composites", Construction and Building Materials, 18, 2004, pp 9-19.

[9] Gálvez, J.C., Cendón, D.A. and Planas, J., "Influnes of Shear parameters on Mixed-Mode Fracture of Concrete", International Journal of Fracture, 118, 2002, pp 163-189.

[10] Hillerborg, A., Modéer, M. and Peterson, P. E., "Analysis of Crack Formation and Growth in Concrete by Means of Fracture mechanics and Finite Elements", Cement and Concrete Research, 6, 1976, pp 773-782.

[11] Bažant, Z.P., "Choice of Standard Fracture Test for Concrete and Its Statistical Evaluation", International Journal of Fracture, 118, 2002, pp 303-337.

[12] Gustafsson, P.J, and Hillerborg, A., "Improvements in Concrete Design Achieved Through the Application of Fracture Mechanics", Application of Fracture Mechanics to Cementitious Composites, Evanston, USA, 1984, pp 639-680.

[13] Guinea, G.V, Planas, J. and Elices, M., "A General Bilinear Fit for the Softening Curve of Concrete", Materials and Structures, 27, 1994, pp 99-105.

[14] Reinhardt, H.W., "Crack Softening Zone in Plain Concrete Under Static Loading", Cement and Concrete Research, 15, 1985, pp 42-52. 